\title{
Trust in translation
}

Cite as: CMAJ 2020 September 28;192:E1136-7. doi: 10.1503/cmaj.201499

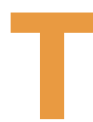

he urgent "click-clack" of my burgundy oxfords reverberates against the sterile white walls of the examination room. I shut the door behind me; the sight ahead is familiar: a worried and concerned family. Her straight, shoulder-length black hair and emerald-green jadeite bracelet alongside his youthful posture and neatly tucked-in polo. Each cradles a baby boy. I look closer, noting thick, semi-torn calluses scattered across his palms and puffy, restless bags drooping from her eyes. They remind me of my parents. I remember hearing about how difficult life was when they immigrated from China in the aftermath of the Cultural Revolution. I am curious about this family's life and have many personal questions, but I hold myself back: I must keep a professional working relationship. Given their last names, I briefly wonder if they would prefer to speak Mandarin, but realize the risk of potentially jeopardizing our relationship by making false assumptions about their language capabilities or upbringings. Furthermore, I lack confidence in Mandarin medical terminology, so I begin in English.

"Hello, what can I do for you today?" I ask.

The father describes episodes of excessive vomiting in one of the fourmonth-old boys. I ask questions: how far does the vomit travel and what colour is it? What are the baby's current intakes and outputs? How are his stooling and urination patterns? Communication is difficult, punctuated with exaggerated hand and arm gestures, Internet images and reframing. All are acknowledged by a flurry of head nods that I cannot interpret. I feel it is not appropriate to conduct a physical examination before gaining the trust of the parents.

Alas, this scenario is so familiar. My parents struggled at first, adapting to

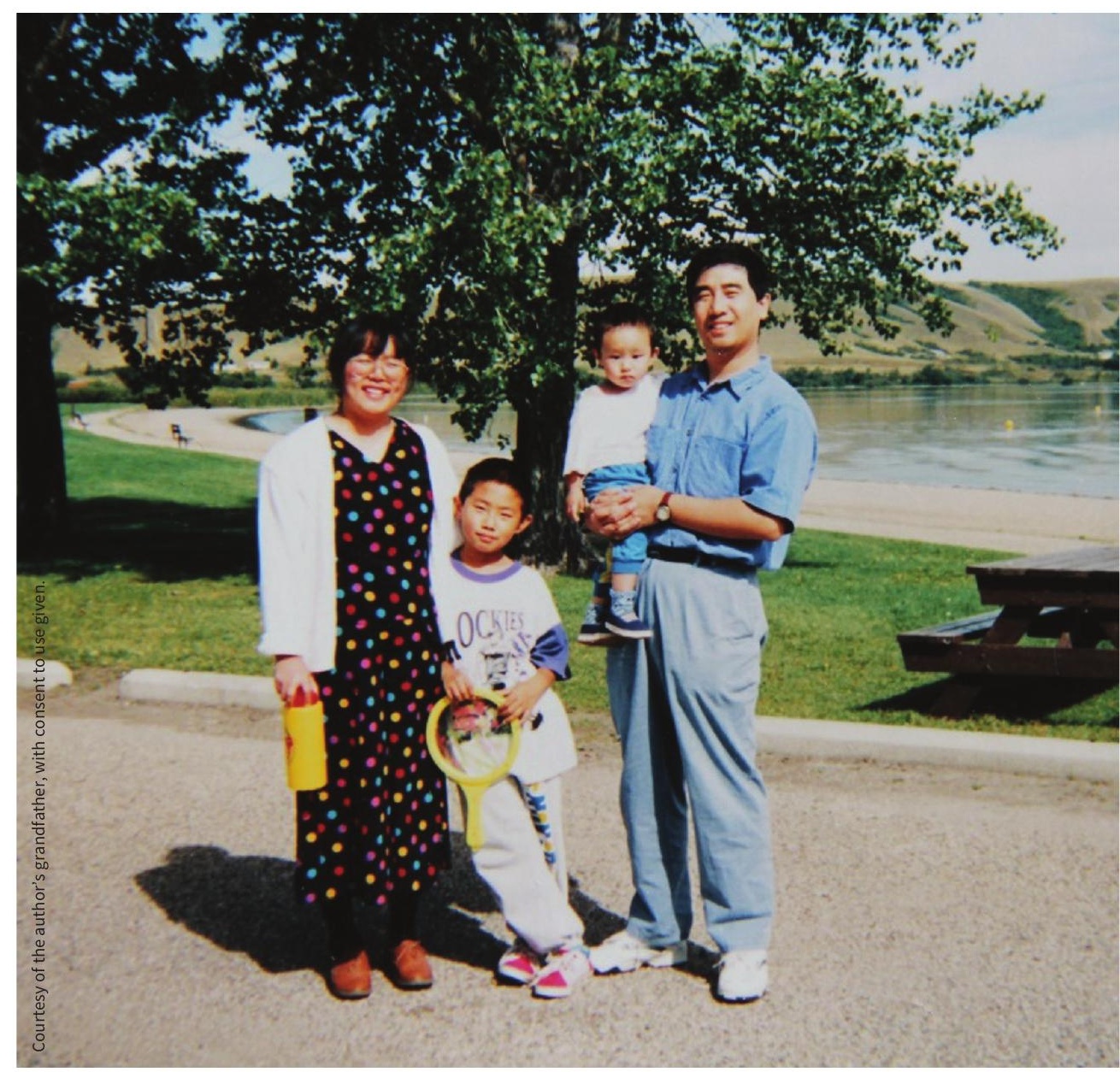

Yang Family, summer 1996 in Regina, Sask.

life in Canada as they moved from being respected academic professors to working multiple minimum-wage jobs to support our family. Difficulties with English plagued every aspect of their lives, from job interviews to creating friendships to asking for help at the grocery store. Language, for many immigrants, is a particularly endemic barrier to a health care system that is already novel and complex.

I feel that my experience, my knowledge, should help me better understand this family's situation.

Tick-tock.
Time is passing. I picture the final grains of sand flowing through an hourglass; our therapeutic alliance is likewise nearing its end, yet we were getting nowhere. In their frustration, they begin speaking between themselves in the language of my childhood; I recall scenes where my parents too could not find the English words, and the phrase comes out of my mouth as if by its own volition.

\section{“对不起, 您想说普通话吗?”}

Translation: Sorry, would you prefer to speak in Mandarin? 
They gaze directly at me in unison. I realize the innate connection I feel is reciprocated. Their eyes widen for a brief moment and we converse eagerly in Mandarin for the rest of the visit. Their bodies angle toward me, their arms uncross. They lean in, listening intently to my questions, newfound comprehension evident on their faces. They indicate that their son is having what sounds like nonbilious projectile vomiting. This prompts me to take a set of vitals and I notice mild tachycardia and moderate dehydration. The abdominal exam is benign, negative for the olive-shaped mass I was palpating for. As I discuss the rare possibility of pyloric stenosis, the atmosphere of trust is as tangible in the air as the parents' measured relief. I explain to the family that urgent blood work, imaging and potential surgical correction may be necessary. Language is a literal and figurative vessel for familiarity, trust and understanding - the underpinnings of a sturdy therapeutic alliance. It ultimately paves the way for a potentially critical diagnosis of pyloric stenosis.

My parents searched for a Mandarinspeaking physician in Regina, Saskatchewan, in the 1990s. This preference was not only for the obvious communication benefits, but also for the understanding and relatability in customs, backgrounds and culture. Having a discussion, even if only small talk about lunar festivity plans, hobbies and interests, or even food recipes, would have made my parents feel more comfortable and laid a stronger groundwork for a therapeutic relationship. This is not to say people of different cultures cannot provide excellent care, but there is an unmet preference for many immigrants for providers of similar language and culture that would provide even better care.

I present this case to my preceptor, who agrees with the plan, and we book the family for the relevant workup. As they leave the clinic, the mother clasps my hands and thanks me profusely while wishing me all the best in my medical studies. I smile, nod and see the family off.

Before this encounter, I had seen countless under-served monolingual Mandarin patients in Vancouver, British Columbia, when I worked in psychiatry at an emergency department. Many were there because of their suicidal ideation. Many disclosed to me that it was not socially acceptable to speak of mental health in their cultures; they had no social supports to fall back on, as their friends and families could not relate. Their previous bids for psychiatric help late at night in the emergency department were plagued with communication difficulties; their struggle to speak English led to repeated misunderstandings and, ultimately, mistrust. These patients explained that the nuances of being able to convey their feelings in their own language mattered deeply, and that by speaking to me they were able to obtain the hospital admission and inpatient psychiatric help they so desperately needed. One particular patient thought that if he sought out psychiatric help, the details would be relayed to his employers and that his job would be in jeopardy. Understanding his unjustified fears ultimately made the difference. I also noted that although translators may be used for communication difficulties, they are not always readily available, and patients may find them less personal than having direct interaction with their physician. In addition, many languages have words that lack a cogent English translation, particularly words relating to feelings and abstract concepts. These lead, inevitably, to losses in translation.

I wondered how I might have done better in that day's encounter. I could have inquired about speaking in Mandarin initially, but this question reveals assumptions about patients' language capabilities, preferences and upbringing. I dislike being asked if I'd prefer to speak in Mandarin purely based on my name and outward appearance; I was born in Canada and am fluently English speaking. I was trying to avoid making this sort of assumption. A previsit question either in triage or via email may help mitigate this.

As new immigrants in the $1990 \mathrm{~s}$, my parents were sometimes thought of as peculiar when they spoke about their food preferences; they were ostracized from social circles because of religious beliefs. Much progress in cultural understanding has been made in the last 30 years, but I believe the best that health care providers can do when caring for diverse cultural populations is to embrace humility. In humility, we will welcome an open and nonjudgmental approach to care, and an understanding and recognition of potential cultural biases.

\section{Yue Bo Yang BSc}

MD Undergraduate Program, University of British Columbia Faculty of Medicine, Vancouver, BC

This article has been peer reviewed.

Specific details of the patient encounter have been altered to conceal patient identity. 